\title{
Originalia
}

Johannes Endler*

\section{Alternative Spiritualität zwischen Nabelschau und sozialem Engagement}

\author{
Alternative spirituality between navel-gazing and social commitment
}

https://doi.org/10.1515/spircare-2018-0054

Zusammenfassung: Einer verbreiteten These folgend wird von alternativ-spirituellen Akteuren keine Änderung der gesellschaftlichen Verhältnisse angestrebt, wesentlich sei hingegen die Arbeit an der eigenen Identität im Dienst der „Selbstverwirklichung“ (vgl. Lasch 1980, List 1988, Bellah et al. 1996). Die Verantwortung für Probleme und deren Lösungen würden dem Einzelnen aufgebürdet und die komplexen Zusammenhänge von (scheinbarer) Selbstbestimmung und internalisierten gesellschaftlichen Ansprüchen blieben verdeckt. Das heutige spirituelle Feld sei marktförmig strukturiert und statt gemeinschaftlicher Beziehungsformen würden flüchtige Begegnungen zwischen unabhängigen Transaktionspartnern vorherrschen (vgl. Hero 2010). Die Individualisierungsthese (vgl. Beck 1986, Beck \& Beck-Gernsheim 2002) bildet den theoretischen Bezugsrahmen für diese oftmals als Kritik vorgebrachten Annahmen. Anhand der für die alternative Spiritualität wichtigen Ansätze von Erich Fromm und Marylin Ferguson werden die beschriebenen Ansichten in der vorliegenden Arbeit relativiert. Inwiefern die in den exemplarischen Selbstzeugnissen formulierten Ansprüche tatsächlich eingelöst wurden und auch für das heutige alternativ-spirituelle Milieu handlungsleitend sind, kann Gegenstand weiterführender Forschungen sein.

Schlüsselwörter: Alternative Spiritualität, Individualisierung, spirituelle Gesellschaftskritik, Erich Fromm, Marilyn Ferguson

\begin{abstract}
Following a widespread assumption, alternative-spiritual proponents do not strive for social change, instead self-realization through working on one's identity is essential (cf. Lasch 1980, List 1988, Bellah et al. 1996). Problems and their solutions would be seen as each individual's own responsibility and complex relations between (apparent) self-determination and internalized social re-
\end{abstract}

*Korrespondenzautor: Johannes Endler, Wien,

E-Mail: endlerjohannes@gmail.com quirements stay concealed. The contemporary spiritual field would be structured on a market basis and contacts between independent partners prevail rather than collaborative relations (cf. Hero 2010). The thesis of individualization (cf. Beck 1986, Beck \& Beck-Gernsheim 2002) serves as a theoretical framework for these (critical) assumptions. The view mentioned is put into perspective on the basis of Erich Fromm's and Marylin Ferguson's approaches considering both as important figures in the field of alternative spirituality. Further research can aim to clarify whether their pretensions are actually met by the alternative-spiritual milieu of their time and today.

Keywords: Alternative spirituality, individualization, spiritual social criticism, Erich Fromm, Marilyn Ferguson

\section{Einleitung}

In der vorliegenden Arbeit werden zunächst die Begriffe „Individualisierung“ und „alternative Spiritualität“ geklärt. In der Fachliteratur werden alternativ-spirituelle Akteure oftmals als auf die eigene Entwicklung und Selbstverwirklichung fixierte Subjekte betrachtet, die Problemursachen und -lösungen dem Individuum - und nicht der Gesellschaft - zuschreiben. Danach werden Ansätze dargestellt, welche diese Ansicht zu relativieren vermögen: erstens Werke des deutsch-US-amerikanischen Psychoanalytikers, Philosophen und Sozialpsychologen Erich Fromm (1903-1980), dessen Menschenbild als exemplarisch für Teile der alternativ-spirituellen Szene gelten kann, und zweitens „The Aquarian Conspiracy“ (1980; dt.: Die sanfte Verschwörung 1982), ein Klassiker der New-Age Literatur der US-amerikanischen Schriftstellerin Marilyn Ferguson (1938-2008). Die Diskussion dazu beinhaltet eine anthropologisch-theologische Perspektive auf Fromms Denken und einen metapherngeschichtlichen Exkurs über Fergusons Netzwerk-Begriff. Überlegungen zu den Verflechtungen der alternativen Spiritualität und des politisch linksalternativen Milieus zu Fergusons und Fromms 
Zeit, Beispiele für aktuelle Initiativen in den Fußstapfen der beiden sowie ein Ausblick auf weiterführenden Forschungsbedarf bilden den Abschluss des Artikels.

\section{Individualisierung}

Der Begriff der Individualisierung wurde vor allem durch die Arbeiten Ulrich Becks (1986, sowie Beck \& Beck-Gernsheim 2002) bekannt und ist - wenngleich ihm Mehrdeutigkeit und mangelnde Präzision angelastet wird (Burkhart 1998: 129) - ein zentraler Bezugspunkt sozialwissenschaftlicher Gegenwartsanalyse. Gemeint ist damit ein die späte Moderne kennzeichnender Prozess, in dem das Individuum zunehmend selbst für seine Biografie zuständig gemacht wird und aus verschiedenen Optionen auswählen muss. Durch die Pluralisierung von Werten und Lebensformen muss sich das Individuum ständig neu positionieren und folgenreiche Entscheidungen treffen, statt sich auf gewohnte Bahnen verlassen zu können. Individualisierung kann als Prozess der zunehmenden Selbstbestimmung verstanden werden, der bestimmte sozialstrukturelle und kulturelle Bedingungen voraussetzt (z.B. Pollack \& Pickel 1999). Man kann damit jedoch auch einen Modus der $\mathrm{Zu}$ rechnung bezeichnen, ein institutionell verankertes „Deutungsmuster, das Selbstkontrolle, Selbstverantwortung und Selbststeuerung akzentuiert“"(Wohlrab-Sahr 1997: 28).

In der vorliegenden Arbeit meint Individualisierung die Praxis,

das Leiden des Einzelnen an der Familie, an den Umständen, an der Gesellschaft in ein Leid zu transformieren, das dem Einzelnen als persönliche Idiosynkrasie, als Makel der Persönlichkeitsentwicklung, als biografische Fehlentscheidung oder als Mangel an Veränderungsbereitschaft zugerechnet werden kann (von Kardorff 2016: 277).

Den Herausgebern des Sammelbandes „Therapeutisierung und Soziale Arbeit“ (Anhorn \& Balzereit 2016) folgend, dem dieses Zitat entstammt, bleiben gesellschaftliche Konstellationen dabei systematisch ausgeblendet. Diese gesteigerte Aufmerksamkeit auf den Einzelnen gehe mit einer zunehmenden Therapeutisierung des Alltags einher (von Kardorff 2016; vgl. auch Maasen 2011; Tändler \& Jensen 2012). Darunter wird verstanden, dass Perspektiven und Praktiken, die ursprünglich einem klinisch-therapeutischen Setting entstammen, heute einen selbstverständlichen Bestandteil nahezu aller gesellschaftlichen und privaten Bereiche bilden. Das Feld der (psychosozialen) Gesundheit und Krankheit werde von verschiedenen miteinander konkurrierenden Akteuren besetzt, welche zu dieser Entwicklung beitragen. Dazu gehören auch esoterisch-spirituell- neureligiöse Formen der Heilpraktik, „die gleichwohl in die ökonomischen Verwertungszusammenhänge und die ideologische Funktionslogik des therapeutisch-industriellen Komplexes gut und fest integriert" sind (Anhorn \& Balzereit 2016: XVIII). Mit dem Dispositiv der Therapeutisierung würden gesellschaftliche Widersprüche als Gegenstand möglichen kollektiven Handelns aus dem Blick geraten und zu psychologisierbaren Fragen nach der eigenen Identität werden. Dabei werde die eigene Lebensführung im Namen nicht genutzter individueller Potenziale und der Verweigerung gesellschaftlich als vernünftig geltender Entscheidungen moralisiert. Bemerkenswert sei dabei,

dass sich ,heteronome (Fremd-)Führung ' und ,autonome (Selbst-)Führung، bis zur Ununterscheidbarkeit miteinander verquicken: Anstelle direktiver und demonstrativer, autoritär-hierarchischer Vorgaben (des Sollens und Müssens) treten vermehrt deutlich zurückgenommenere und auf den ersten Blick weniger als Macht und Herrschaft ersichtliche Formen der Verhaltenssteuerung (Anhorn \& Balzereit XIXf.)

auf. Die Akteure haben so den Eindruck, aus eigenem freiem Antrieb handeln zu können. Im Anschluss an Foucault wird Therapeutisierung zu einem Element einer neoliberalen „Regierung“ und Formierung des Subjekts, bei dem „Anpassungs- und Unterwerfungsleistungen [...] i.d.R. vermittelt (indirekt) und unter aktiver (freiwilliger) Beteiligung der Herrschaftsunterworfenen erzeugt werden “ (Anhorn \& Balzereit: 13). Neue und zum Teil radikale Varianten „freiwilliger“ Selbstoptimierung und Vervollkommnung des Individuums werden heute im Kontext des Trans- und Posthumanismus diskutiert, bei dem die Grenzen menschlicher Möglichkeiten überdies durch technologische Verfahren erweitert werden sollen (Krüger 2010).

\section{Alternative Spiritualität}

Mit der Säkularisierungsthese verbundene soziologische Ansätze teilen die Behauptung, Religion verliere in den sich modernisierenden Gesellschaften an sozialer Bedeutung. Sie habe keine klare Kontur und könne keine für die Gesellschaft im Ganzen verbindlichen Deutungen und kollektiven Werte mehr bereitstellen. Dabei wird ein fundamentales Spannungsverhältnis von Religion und Moderne angenommen (Berger 1973; Martin 1978; Wilson 1982; Tschannen 1991; Bruce 2002, Dobbelaere 2002; Pollack 2003; Pollack \& Rosta 2015). In kritischer Auseinandersetzung mit dieser Sichtweise wurde der Begriff Individualisierung - im Sinne Becks - in den letzten Jahrzehnten zu einer zentralen (religions-)soziologischen Kategorie (Luckmann 1967/1991; Pickel 2011). Von einem empirisch 
beobachtbaren Rückgang der Kirchlichkeit könne man nicht ohne weiteres auf ein Verschwinden der Religiosität per se schließen. Eher sei von einer Transformation des Religiösen zu sprechen, von unterschiedlichen Formen subjektiver bzw. individueller Religiosität. Diese unsichtbare Religion (Luckmann 1991) finde sich auch jenseits der etablierten Kirchen und könne oft nicht ohne weiteres in einem herkömmlichen Sinn als religiös identifiziert werden. Gemäß dem Paradigma der religiösen Individualisierung gibt es keinen einheitlichen, gesamtgesellschaftlich akzeptierten „heiligen Kosmos“ mehr. Stattdessen könnten Interessenten aus dem religiösen Angebot frei wählen und es komme zu synkretistischen Verbindungen und individuellen Systemen letzter Bedeutung. Es sei angemerkt, dass die Suche nach individueller Religiosität freilich weiterhin von neuen lebensweltlichen Mustern strukturiert wird und sich an Werten und Normen orientiert, die sich für den Einzelnen aufgrund der jeweiligen sozialstrukturellen Einbettung als plausibel erweisen (Hero 2010).

Die alternativ-religiöse Szene wird im alltäglichen Sprachgebrauch und in der Selbstbeschreibung heute oftmals als „spirituell“ bezeichnet. Auch wenn dies hinsichtlich der Geschichte des Begriffs der Spiritualität und seiner Bedeutung in verschiedenen rezenten religiösen Traditionen eine Verengung darstellt, wird er auch in sozialwissenschaftlich orientierten Studien zunehmend in diesem Sinn verwendet. Hubert Knoblauch sieht ein zentrales Merkmal der so verstandenen Spiritualität darin, dass persönliche außeralltägliche Erfahrungen wichtig genommen und auf ,symbolische außerweltliche Weise gedeutet werden“ (Knoblauch 2006: 100). Dies treffe in besonderer Weise auf „alternativ-spirituelle Bewegungen“ zu. Darunter versteht Knoblauch das bis in die 1990er Jahre hinein so bezeichnete New Age, das sich aus uneinheitlichen, vorrangig „alternativ-“ religiösen, psychologischen und weiteren kulturellen (etwa populärwissenschaftlichen) Quellen speist. Der von mir verwendete Begriff der alternativen Spiritualität meint das New Age ebenso wie Teile des Human Potential Movement und die „ganzheitlichen“ spirituellen und therapeutischen Aktivitäten der heutigen Selbsterfahrungsszene. Markus Hero sieht neben Individualismus und Erlebnisorientierung einen ausgeprägten Anthropozentrismus (das „Göttliche“ liege im Menschen) und die Diesseitsorientierung (,Sakralisierung des Alltags“) als zentrale Merkmale der „neuen Religiosität“ an (Hero 2010). Die „alternative“ Spiritualität ist heute vielfach Teil des kulturellen Mainstreams, man denke etwa an die breite Rezeption esoterischer oder (vermeintlich) fernöstlicher Praktiken im Wellness-Bereich oder in der Management-Literatur. Kritiker sehen darin eine Tendenz zum Rückzug ins Private und eine schwindende Bereit- schaft, Verantwortung für die Gesellschaft zu übernehmen (vgl. z. B. Lasch 1980; List 1988; Bellah et al. 1996)

Nicht selten wird ein ,anti-institutionalistischer Gestus“ (Knoblauch 2002: 303), eine Tendenz zur „De-Institutionalisierung “ (Pollack \& Pickel 2003) als wichtiges Merkmal der neuen religiösen Szene und insbesondere der alternativen Spiritualität angesehen. Laut Markus Hero (2010) gibt es allerdings keine generelle Abschwächung institutioneller Strukturen, sondern es bestehen weiterhin vermittelnde soziale Gebilde, in denen religiöse Akteure interagieren und die das faktische Ausleben der neuen Religiosität überhaupt erst ermöglichen. Indem er die Entwicklung der neuen Religiosität seit den 1960ern chronologisch nachzeichnet, sieht Hero nach der Gemeinschaft und der Organisation heute den Markt als die primäre Weise ihrer Institutionalisierung an. Dieser sei gekennzeichnet durch eine bestimmte Art der Tauschbeziehung, bei der von einander unabhängige Transaktionspartner flüchtige klientenförmige Beziehungen eingehen, die in der Regel keine Verpflichtung für die Zukunft nach sich ziehen.

Um kurz zu rekapitulieren - bis hierher wurden mehrere Thesen über alternative Spiritualität aufgestellt: Es wird in ihnen keine Änderung der gesellschaftlichen Verhältnisse angestrebt, wichtig ist stattdessen das individuelle Verhalten und die Arbeit an der eigenen Identität im Dienst der Selbstverwirklichung. Die Verantwortung für Probleme und deren Lösungen werden dem Einzelnen aufgebürdet und die komplexen Zusammenhänge von (scheinbarer) Selbstbestimmung und internalisierten gesellschaftlichen Ansprüchen bleiben verdeckt. Es herrschen marktförmige Beziehungen zwischen unabhängigen Transaktionspartnern vor.

\section{Persönliche Transformation = gesellschaftliche Transformation?}

Der nun folgende Hauptteil dieses Artikels widmet sich zunächst der Frage, wie Themen der Individualisierungsthese innerhalb des alternativ-spirituellen Feldes verhandelt werden. Dabei geht es insbesondere darum, ob und gegebenenfalls wie Problemursachen und -lösungen dem Individuum oder der Gesellschaft zugeschrieben werden, sowie um das Zueinander von Selbst- und Fremdbestimmung und den Anspruch auf Selbstverwirklichung. Mitberücksichtigt wird dabei die Frage nach der zugrunde liegenden Anthropologie hinsichtlich der Beziehung zwischen dem Individuum und seiner sozialen Umwelt.

Als primärer Bezugspunkt dient exemplarisch das Werk Erich Fromms, der durch Bestseller wie „Die Kunst 
des Liebens“ (1956) und „Haben oder Sein“ (1976) zu einem bedeutenden „public intellectual“ (Jacoby 1987) wurde. Fromms Beitrag zur Entstehung der Kritischen Theorie der Frankfurter Schule wird heute hingegen weitgehend ignoriert, was nicht zuletzt auf ein Zerwürfnis mit seinem früheren Kollegen Herbert Marcuse zurückzuführen ist. Marcuse, ein Idol der linken Studentenbewegung, warf Fromm unter anderem vor, er verwandle soziale Unterdrückung in ein moralisches Problem und mache das Individuum dafür verantwortlich, dass es seine Möglichkeiten nicht voll entfalte (Marcuse 1969; zum Verlauf der Fromm-Marcuse Debatte vgl. Rickert 1991 und McLaughlin 2017). Marcuses Kritik steht im Einklang mit der generellen Einschätzung des „Psychobooms“ und der Spiritualität durch Teile der Linksalternativen, wenngleich die Szene der Neuen Spiritualität und des linksalternativen Milieus in den 1970er Jahren beträchtliche Berührungspunkte aufwies (Reichardt 2014). Fromms religionspsychologische Schriften wurden unter dem Aspekt ihrer jüdischen, buddhistischen und religionskritischen Prägung gedeutet (z. B. Hardeck 1992; Domagoj 2011). Zudem spielte er eine wichtige Rolle in der Geschichte der alternativen Spiritualität. So beeinflusste er das Human Potential Movement der 1960er Jahre, welches wiederum das New Age der 1980er Jahre befruchtete und heute in der spirituellen Selbsterfahrungsszene weiterlebt. Diese Entwicklung wurde und wird von der Internationalen Erich-Fromm-Gesellschaft kritisch gesehen:

\footnotetext{
„Wir haben also die Situation, dass die außerakademische Frommrezeption (während die akademische seit langem stagniert) über einen Teilmarkt abgewickelt wird, auf dem die entsprechenden Titel unmittelbar mit den Esoterik-Autoren konkurrieren“ (Klein-Landskron 1990: 3; in den letzten Jahren ist hingegen wieder ein steigendes akademisches Interesse an Fromm zu beobachten, vgl. McLaughlin 2017: 8f. mit Literaturhinweisen).
}

Eine vertiefende Untersuchung der Fromm-Rezeption in esoterikaffinen Spiritualitätskreisen steht laut Fromms Nachlass- und Rechteverwalter Rainer Funk bislang noch aus (E-Mail an den Autor, 3.10.2018) und wird auch in dieser Arbeit nicht geleistet.

Danach wird skizziert, welche institutionellen Formen die Akteure selbst als geeignet für die Verwirklichung ihrer Ziele halten. Hierfür wird der Bestseller „Die sanfte Verschwörung“ (1982) von Marilyn Ferguson als Beispiel herangezogen. Wouter Hanegraaff zufolge enthält dieses „New-Age-Manifest“ (Hanegraaff 1998: 106) charakteristische Positionen des frühen New Age der 1960er und 1970er Jahre, wobei er nicht die verbreitete Ansicht teilt, Ferguson sei repräsentativ für die gesamte „Bewegung“ inklusive der Entwicklungen der 1980er Jahre. Der in unserem Kontext relevante Begriff des „Netzwerks“, wie ihn Ferguson verwendet, ist m. E. in der heutigen alternativen Spiritualität jedoch weiterhin aktuell, wie unten noch deutlich werden wird.

\section{Erich Fromms humanistische Religion als Beispiel alternativer Spiritualität}

Fromms (religionspsychologische) Arbeiten enthalten selbst kein geringes religionsproduktives Moment. Beispielhaft dafür ist „Zen-Buddhismus und Psychoanalyse“ (1960/1971, Zitation im Folgenden nach der neueren Ausgabe), das unter anderem einen Text von Daisetz Teitaro Suzuki (1870-1966), einem der bedeutendsten Zen-Lehrer im Westen, enthält. Fromm und Suzuki stehen jeweils für eine besondere Interpretation der Psychoanalyse und des Zen-Buddhismus und wollen eine Brücke zwischen östlicher Religiosität und westlicher Wissenschaft schlagen. Dieses Thema wurde in religiösen Reformkreisen bereits um 1900 diskutiert (vgl. z. B. Vivekananda, 1893/1935) und blieb für die spätere New Age Bewegung zentral (vgl. z. B. Capra 1992). Die Praxis des Zens ist für Suzuki die wissenschaftliche Religion der Zukunft. Es handle sich um eine originär japanische und zugleich universale Erfahrung, um die Essenz des religiösen Bewusstseins der Menschheit (Borup 2004). Suzuki wird dabei einerseits von einem mit westlicher Aufklärungsphilosophie vertrauten (nationalistischen) Reformbuddhismus, andererseits von „esoterischen“ europäisch-amerikanischen Denkern beeinflusst (Borup 2004).

Psychoanalyse und Zen beschäftigen sich nach Fromm mit dem Wesen des Menschen und mit einem Weg, der zu seiner Wandlung führt. Dabei sieht er einige Ansätze des Zen in Sigmund Freuds Denken bereits implizit angelegt, die er in seiner eigenen humanistischen Psychoanalyse schließlich entfaltet. Grundlegend für Fromms Denken ist sein Verständnis des Menschen, welches er auch in seinem Aufsatz über Zen darlegt. Der Mensch sei damit konfrontiert, dass er nicht wie das Tier in seine Umwelt eingepasst sei. Er sei Teil der Natur, gehe aber zugleich über sie hinaus, indem er Kraft der Vernunft ein Bewusstsein seiner selbst entwickle. Er müsse sein Leben selbst leben und werde nicht von außen gelebt. Dies führe ihn zu der Erfahrung von Einsamkeit und Hilflosigkeit. Jeder, für den es eine Angelegenheit von höchster Wichtigkeit ist, sich dem Problem seiner Existenz als ganzer Mensch zu stellen, ist für Fromm ein religiöser Mensch. Die Psychoanalyse, so wie Fromm sie versteht, wird so implizit als religiöses Unterfangen charakterisiert. Die Über- 
windung der Isoliertheit könne nun - auf der Ebene des Individuums und auf der Ebene religiöser Systeme durch den zum Scheitern verurteilten Versuch der Rückkehr in den Zustand der Harmonie vor der Geburt bzw. in der tierischen Seinsweise bestehen. Die andere Antwort laute, ganz geboren zu werden,

das Bewußtsein, die Vernunft, die Fähigkeit zu lieben bis zu einem Grad zu entwickeln, daß man seine eigene egozentrische Einbezogenheit hinter sich läßt und zu einer neuen Harmonie, einem neuen Einssein mit der Welt gelangt (Fromm 1960/1971: 113f),

wobei man sich dennoch gleichzeitig als separate Ganzheit erlebt. Das beinhaltet einen aktiven, schöpferischen Zugang zur Welt, die ohne Verzerrungen so gesehen wird, wie sie ist.

Laut Fromm befindet sich die Gesellschaft der Gegenwart in einer Krisensituation. Der Mensch habe sich in ein Ding verwandelt und das Leben dem Eigentum untergeordnet. Er habe die Illusion eines väterlichen Gottes als Helfer fallen lassen, aber auch die Ziele der humanistischen Religion:

die Überwindung der Grenzen, die ein egoistisches Ich setzt, die Verwirklichung von Liebe, Objektivität und Demut und die Ehrfurcht vor dem Leben, die als Ziel des Lebens das Leben selbst sieht und den Menschen zu dem macht, was er seinen Anlagen nach sein kann (Fromm 1960/1971: 104).

Fromm sieht diese Ziele, die auch ein Ideal des heutigen spirituellen Feldes sind, im Zen auf herausragende Art verwirklicht. Doch statt diese dem Menschen angemessenen, „wahren“ Werte zu fördern, sei das heutige Denken von gesellschaftlich bedingten Fiktionen und Täuschungen bestimmt.

Während des größten Teils der Geschichte der Menschheit hat stets [...] eine kleine Minderheit über die Mehrheit ihrer Mitmenschen geherrscht und sie ausgebeutet. [..] Auf die Dauer mußte die Mehrheit ihre eigene Ausbeutung freiwillig anerkennen und das ist nur möglich, wenn ihr Geist mit den verschiedensten Lügenmärchen erfüllt wurde, die die Anerkennung der Herrschaft der Minderheit erklärten und rechtfertigten (Fromm 1960/1971: 126).

Jede Gesellschaft forme über die Vermittlung der Familie den „Gesellschaftscharakter“ ihrer Mitglieder so, „daß sie das tun wollen, was sie tun müssen“ (ebd.: 133). Den zumeist negativen Einflüssen der Gesellschaft stellt Fromm das dem Menschen inhärente Streben nach Entwicklung gegenüber. Die Biophilie, die Liebe zum Lebendigen, sieht Fromm als primäres Prinzip, die Nekrophilie - sowie verschiedene nicht-produktive Charakterorientierungen - als „Folge eines gehemmten Wachstums“ (Fromm 1974: 411f.). Die Annahme einer solchen Lebensbejahung entspricht weniger der psychoanalytischen Orthodoxie. Sie ist aber zentral für die humanistische Psychologie und die alternative Spiritualität, wo man sie dem „inneren“, „höheren“ oder „wahren Selbst“ zuschreibt. Im besten Fall wären demzufolge die Ziele der Gesellschaften deckungsgleich mit den Zielen des Menschen bzw. der Menschheit als Ganzes.

Es gelte, so Fromm, die Wechselbeziehungen zwischen ökonomischen und geistigen Ursachen für den $\mathrm{Zu}$ stand einer Gesellschaft zu sehen.

Das Christentum hat die spirituelle Erneuerung gepredigt und darüber die Veränderung der Gesellschaftsordnung verabsäumt [...], der Marxismus hat die Notwendigkeit sozialer und wirtschaftlicher Veränderungen in den Vordergrund gestellt und dabei die Notwendigkeit einer inneren Wandlung des Menschen übersehen (Fromm 1955/2003: 230).

Ein produktiver Gesellschafts-Charakter könne nur in einer Gesellschaft ermöglicht werden ,in welcher kein Mensch für einen anderen Mittel zum Zweck ist, sondern in der er stets und ausnahmslos Selbstzweck ist“ (ebd.: 234). Alle ökonomischen und politischen Tätigkeiten müssten dem Ziel des inneren Wachstums des Menschen untergeordnet sein.

Nach Fromm führt die vernunftbedingte Fähigkeit des Menschen zur Distanzierung von sich selbst zu einer problematischen Situation. Der Mensch empfinde seine Existenz als unsicher und fragwürdig und suche Zuflucht bei Religionen, um mit dieser Situation - im Sinne einer Kontingenzbewältigung - umzugehen. An anderer Stelle bezeichnet Fromm Religion als ,jedes von einer Gruppe geteilte System des Denkens und Handelns, das dem Einzelnen einen Rahmen der Orientierung und ein Objekt der Hingabe bietet“ (1976/2010: 365f.). Der Existentialismus Jean Paul Sartres, auf den sich Fromm nicht explizit bezieht, kommt, ausgehend von der Weltoffenheit des Menschen, zu einer Absage an essentialistisch gefasste Ordnungen und Weltanschauungen sowie an einen Gottesglauben. Für Fromm macht hingegen gerade die beschriebene Wesensbestimmung des Menschen die Religion notwendig. Sie wird so einerseits funktionalistisch gedeutet, andererseits ist sie eine unumgängliche Reaktion auf ein existenzielles menschliches Bedürfnis (Kolbe 1986). Die Frage ist für Fromm demnach wie oben angedeutet nicht, ob jemand religiös ist, sondern auf welche Art und Weise. Die für den Menschen förderliche humanistische Religion sieht nun, wie im Vorhergehenden beschrieben, das Leben selbst als Ziel des Lebens und beansprucht, den Menschen zu dem zu machen, was er seinen Anlagen nach sein kann. Fromm be- 
zeichnet die religiöse Haltung als $X$-Erfahrung und meint damit das

Aufgeben des Wunsches, sich an das „Ich“ zu klammern, als ob dieses ein unzerstörbares, separates Gebilde wäre; als ein Leerwerden, um sich mit „Welt“ füllen zu können, um auf sie zu reagieren, mit ihr eins zu werden, sie zu lieben. Leer werden ist kein Ausdruck von Passivität, sondern von Offenheit (Fromm 2008: 51).

Der Aufstieg zu neuer Harmonie bedeutet nach Fromm eine Selbsterlösung durch die Vernunft, welche zugleich für die widersprüchliche Situation des Menschen verantwortlich ist. Gott wird demnach zu einem Symbol für die Fähigkeit des Menschen, zu einer höheren Stufe der Selbsterkenntnis und der Liebesfähigkeit zu gelangen, zu seinem wahren Selbst zu finden (vgl. auch Höllinger \& Tripold 2012).

Die Ansicht, die Verwirklichung der höchsten Anlagen sei das Ziel des Menschen, findet sich bereits prominent bei Aristoteles (vgl. Nikomachische Ethik I, 5) und ist heute ein Grundgedanke der humanistischen Psychologie (vgl. Remele 2001), der modernen Esoterik (vgl. Runggaldier 1996) und der alternativen Spiritualität. Selbstverwirklichung wird seitens der Befürworter mit persönlichem Wachstum, Kreativität und Treue zu sich selbst assoziiert, mit Betonung des Gefühls gegenüber einseitigem Intellektualismus, mit Protest gegen Fremdbestimmung, institutionellen Zwängen und Rollenerwartungen (Remele 2001). Kritiker wie Christopher Lasch (1980) meinen, unter Berufung auf Selbstverwirklichung bzw. Authentizität werde alles außer Acht gelassen, was über das Ich und seine momentanen Befindlichkeiten hinausgehe. Charles Taylor (1996) gibt der Kritik an einer rein selbstbezogenen Selbstverwirklichung recht (vgl. Assing-Hvidt et al. 2012). Allerdings nimmt er das Anliegen der Selbstverwirklichung ernst und versucht es besser zu verstehen. Er geht davon aus, dass Bildung und Aufrechterhaltung der eigenen Identität zeitlebens dialogisch verlaufen. Nur im Kontakt mit anderen, die uns etwas bedeuten, lernten wir uns selbst kennen bzw. formten wir überhaupt erst unser Selbst. Jede zustimmungswürdige Selbstverwirklichung müsste dieser Dimension der Begegnung und dem immer mitgegebenen ,geteilten Bedeutungshorizont“ (Taylor 1996: 47) Rechnung tragen.

Gunda Schneider-Flume (1978) arbeitet hingegen heraus, dass Fromm von einem primär sprachlos-isolierten Vernunftwesen ausgeht, das nur auf sich selbst bezogen ist und sich ein Verhältnis zur Welt und den Mitmenschen erst schaffen muss. Bei Fromm muss die ursprüngliche Vereinzelung überwunden werden, um geistig gesund zu werden. Im Unterschied zu einer theologischen Anthropologie stehe der Frommsche Humanismus zudem unter einem ungeheuren Zwang:
Der Mensch muß leben [...] er muß sich erschaffen und so den Sinn seines Lebens produzieren, sonst verfehlt er das Leben. Das Leben des Menschen, wie Fromm es versteht, ist gekennzeichnet durch den Verlust der Dimension des Dürfens. Leben ist für Fromm nicht eine Gabe, über die man sich freuen kann und für die man dankbar sein kann; anfänglich ist Leben Fluch, der Mensch selbst muß diesen Fluch in Segen umschaffen (Schneider-Flume 1978: 144).

Die Überwindung der Isolation führt bei Fromm nun über die sogenannte „produktive Liebe“ (Fromm 1956/2003: 36). Diese wird nicht im Sinne des Freudschen Sexualinstinkts oder als großes Gefühl im Sinne der These der Erlebnisgesellschaft verstanden, sondern als ethische Tat, die ein Leben lang geübt wird, gerade gegenüber den Hilflosen, Armen und Fremden. Fromm (1974/1999) versteht die Aktivität des Liebens als Geben, als Phänomen des Überflusses und Reichtums der Persönlichkeit. Wieder verweist Schneider-Flume darauf, dass das Geben hier ganz vom Subjekt, vom sich selbst produzierenden Individuum her verstanden wird, und stellt die Frage, „ob dieser Mensch den Kreis seiner Selbstverwirklichung überhaupt transzendieren kann, ob sein Geben überhaupt den anderen erreicht" (Schneider-Flume 1978: 149). Nächstenliebe sei bei Fromm davon bestimmt, dass ich mich selbst verwirkliche, nicht davon, dass der andere etwas braucht.

Die konkrete Person des Nächsten wird durch den „zwanghaften Rückbezug auf das Problem der Selbstverwirklichung“ (ebd.: 151) laut Scheider-Flume gar nicht richtig wahrgenommen und erscheint lediglich unter dem Aspekt der „Identität des menschlichen Kerns, der allen Menschen gemeinsam ist“ (Fromm 1956/2003: 71). Wenn man diesen Gedanken mit Emmanuel Levinas weiterverfolgt, vermag Fromm anscheinend nicht, den Anderen als Anderen zu begreifen. Diese Position Fromms ist hier m. E. charakteristisch für Teile der alternativen Spiritualität, welche in der „Ganzheit“ bzw. dem „Holismus“ Grundlage und Ziel ihres Weltbildes sehen. Nach Levinas gibt es in diesem „totalitären“ Denken kein Außerhalb des Subjekts und der von ihm entworfenen Ordnung des Seins. Der Blick des sich so selbst Verwirklichenden sei herrschaftlich, stets darauf aus, mit der Welt ein Ganzes zu bilden und sie so in die eigene Totalität zu integrieren. Außer Acht bleibe hier das Getroffen-Werden vom Blick des Anderen, in dem sich seine „Unendlichkeit“, seine Unfassbarkeit erschließt (Levinas 2002: 110). Im Blick des Anderen trifft mich ein verborgenes Zentrum, ein entzogener Ursprung, dessen ich mich nicht bemächtigen kann - sofern ich vom Bedürfnis nach Ganzheit ablasse. Einige Passagen in Fromms Werk relativieren ein solches Totalitätsdenken. Etwa wenn er vom Einssein mit der Welt schreibt, 
bei dem der Mensch sich doch als separate Ganzheit erlebe (Fromm 1960/1971), oder wenn er in Anlehnung an Immanuel Kant fordert, kein Mensch dürfe für einen anderen als Mittel zum Zweck - und damit auch nicht der eigenen Selbstverwirklichung - dienen (Fromm 1955/2003). Nichtsdestotrotz ist Schneider-Flumes Analyse im Grunde recht zu geben. Sie könnte im Zuge weiterer Forschungen zur alternativen Spiritualität fruchtbar gemacht und weiterentwickelt werden.

\section{Marilyn Fergusons „sanfte Verschwörung“}

Ferguson ist mit ihrem einflussreichen Werk „Die sanfte Verschwörung“ (1982) eine Protagonistin des New Age, das besonders in den 1980er Jahren für die Hoffnung auf ein anbrechendes neues Zeitalter steht. Es handelt sich bei dieser Strömung um eine optimistische Kulturkritik, die sich selbst in Opposition zu den Werten der „alten“ Welt definiert. Fromms Beschreibungen der aktuellen „Krise“ und der Vision von persönlicher und gesellschaftlicher Gesundheit sowie seine Gegenüberstellung von autoritärer und humanistischer Religiosität wird in Abwandlungen in vielen New Age Texten wieder aufgenommen. Neben der Erwartung einer harmonischen, ganzheitlichen Weltordnung, durchaus im Sinne Fromms, gibt es auch radikalere Narrative eines kommenden „Age of Light“ mit ungeahntem Glück und grenzenlosem Bewusstsein (Hanegraaff 1998: 337). Die Menschheit sei jedenfalls an einem entscheidenden Punkt in ihrer Geschichte angelangt. Der Wandel kann als unausweichliches Geschehen verstanden werden, die meisten New Age-Autorinnen und Autoren gehen jedoch davon aus, dass ein aktiver menschlicher Beitrag, insbesondere Arbeit am inneren Selbst, für eine Wendung zum Guten erforderlich ist. Die Annahme, dass eine transformierte Minderheit einen weltweiten Wandel hervorbringen kann, ist dabei weit verbreitet. Sobald eine bestimmte „kritische Masse“ von Menschen mit höherem Bewusstsein erreicht sei, würde die übrige Menschheit in einer Kettenreaktion folgen (vgl. ebd.: 350 f.; dieser Gedanke ist auch unter dem Namen „Prinzip des hundertsten Affen“ bekannt).

Spiritualität ist für Ferguson (1980) die anzustrebende Weiterentwicklung der Religion. Sie versteht darunter ein auf eigener Erfahrung beruhendes direktes Wissen, das sich vom Glauben aus zweiter Hand unterscheidet. Ferguson steht damit in einer angelsächsischen Bedeutungstradition des Ausdrucks „Spiritualität“, die am Ende des 19. Jahrhunderts aufkam und sich von der katholisch-ordenstheologischen Traditionslinie unterscheidet (Bochinger 1994). Spiritualität erlaubt bei Ferguson eine unmittel- bare Wahrnehmung der Natur und damit sei sie der modernen Naturwissenschaft ebenbürtig. Sie führe zu einem kohärenten Verstehen der Wirklichkeit und damit verbunden zu einer ökologischen Ethik, welche helfe, die negativen Folgen der Naturwissenschaften $\mathrm{zu}$ kompensieren (Bochinger 1994).

Ferguson zitiert anerkennend Erich Fromm, demzufolge sich eine Bewegung abzeichne, die den Wunsch nach tiefgreifender gesellschaftlicher Veränderung mit neuen spirituellen Perspektiven verbindet.

\begin{abstract}
Weder Staat noch politische Parteien oder die organisierte Religion könnten [sic] für diesen Vorstoß eine intellektuelle oder spirituelle Heimat bilden [...] Der Schlüssel zum Erfolg der Bewegung bestünde darin, dass sie im Leben ihrer überzeugtesten Mitglieder verkörpert würde [...] Sie würden inmitten der Entfremdung des heutigen sozialen Milieus ihre eigene Welt aufbauen (Ferguson 1982: 65).
\end{abstract}

Ferguson betrachtet das persönliche Beispiel als wichtigsten Faktor für gesellschaftliche Veränderungen und bezieht sich dabei wieder auf Fromm, wonach „keine großartige umwälzende Idee überleben könne, solange sie nicht in Individuen verkörpert wird, deren Leben selbst die Botschaft darstellt. Das transformierte Selbst ist das Mittel dazu“ (ebd.: 136). Der Weg zur Heilung der Gesellschaft führe zunächst über mehr Bewusstsein, insbesondere mehr Bewusstsein des eigenen Körpers, welches sich durch regelmäßig zu übende Körpertechniken erreichen lasse. Allerdings seien das Selbst und die Gesellschaft untrennbar miteinander verbunden. „Irgendwann muß jeder, der sich mit der Transformation des Individuums beschäftigt, zum gesellschaftlichen Handeln übergehen“ (ebd.: 221). Ferguson bezeichnet dies als „Do-it yourselfRevolution“ (ebd.: 71), bei der sich Personen, die eine transformative Vision teilen, in Netzwerken zusammenschließen. Sie beschreibt Netzwerke in allen gesellschaftlichen Bereichen zu Themen wie Medizin, Recht, Verwaltung, Wahnsinn, Kunst, Tod und alternative Geburt, Ökologie, Ernährung und so fort.

\section{„Biophile“ Gesellschaften und spirituelle „Netzwerke“}

Die im Rahmen der Forschungsfragen angesprochene Individualisierung gesellschaftlicher Probleme und das $\mathrm{Zu}$ einander von Fremd- und (scheinbarer) Selbstbestimmung wird von Erich Fromm eingehend behandelt. Selbstbestimmung steht für ihn im Einklang mit einem postulierten, dem Menschen innewohnenden, auf Entwicklung aus- 
gerichteten und lebensbejahenden Prinzip. Verdeckte Fremdbestimmung ist hingegen die unbewusste Übernahme von gesellschaftlich vorgegebenen Werten, welche diesem Prinzip widersprechen. Fromms Ansatz zur Lösung der „gegenwärtigen Krise“ setzt zwei Schwerpunkte. Einerseits sollen auf heilsame Weise die inneren Spannungen aufgedeckt werden, welche sich durch das anthropologisch bedingte reflexive Selbstverhältnis sowie durch den Widerspruch zwischen Gesellschaftscharakter und inneren biophilen Neigungen ergeben. Dies kann durch spirituelle Praxis oder in einer (humanistischen) Psychotherapie geschehen. Andererseits soll die Gesellschaft dahingehend verändert werden, dass sich ein produktiver Gesellschaftscharakter entwickeln bzw. eine humanistische Religion etablieren kann. Eine Diskussion von Fromms Vision eines sozialistischen Humanismus findet sich bei Johach (2008). Fromms übergeordnetes Ziel besteht darin, die inneren Anlagen des Menschen - wie Liebe und Erkenntnis - zur Entfaltung zu bringen. Seitens der politischen Linken im Gefolge Marcuses wird unter anderem kritisiert, dass die Betonung von Wachstum und „Produktivität“ sich nicht vom „Ziel des gesunden Individuums unter dem Leistungsprinzip" (Marcuse 1969: 254) unterscheide und Fromm im Grunde den gesellschaftlichen Status Quo erhalten wolle. Dagegen lässt sich einwenden, dass Fromm auf Basis seiner Anthropologie bestimmte Gesellschaftsordnungen danach beurteilt, ob sie „produktive” Antworten auf die existenziellen Bedürfnisse fördern oder unterdrücken. Das Kriterium für seelische Gesundheit sei „nicht, dass der einzelne an eine bestimmte Gesellschaftsordnung angepasst ist”, sondern es handle sich „um ein universales, für alle Menschen gültiges Kriterium, dass sie nämlich für das Problem der menschlichen Existenz eine befriedigende Antwort finden" (Fromm 1955/2003: 14). Fromms Menschenbild kann von marxistisch-freudianischer Seite als idealistisch und von theologisch-anthropologischer Seite als zu wenig dialogisch problematisiert werden. In seinen praktischen Auswirkungen zielt ein daran orientiertes Handeln jedenfalls auf das eigene Wohl ebenso wie auf das Wohl der Mitmenschen und eine entsprechende Änderung der gesellschaftlichen Verhältnisse.

Marylin Ferguson will dazu beitragen, solche institutionellen Bedingungen zu schaffen, die zur Erreichung persönlicher und gesellschaftlicher Transformation nötig sind. Die Rede ist von einem „Netzwerk“ spirituell und sozial engagierter Menschen. Wie Friedrich \& Biemann (2016) in ihrer begriffs- und metapherngeschichtlichen Studie zeigen, tritt das Konzept des Netzwerks - im Vergleich zu früheren Konnotationen des Netz- bzw. Netzwerkbegriffs - spätestens ab den 1980er Jahren mit einem normativen, sozialutopischen Gehalt auf. Man versteht darunter die Selbstorganisation und Kooperation autonomer Individuen, die durch ihre Interaktionen Netzwerke hervorbringen, in denen es keinen zentralistischen Ort der Machtausübung mehr gibt bzw. geben soll. Netzwerke gelten nun als ideales Modell demokratischer Kollektive (Friedrich \& Biemann 2016: 5).

Im Kontext des New Age erlangt der normative Anspruch sozialer Netzwerkkonzeptionen eine naturalistische Legitimation - „Wenn sich die Natur in Netzen organisiert, werden wir umso mehr in Einklang mit ihr stehen (und also besser leben), wenn wir es ihr nachtun“ (ebd.: 5). Ferguson propagiert das network in diesem Sinn als ein „tool for the next step in human evolution“ (1980: 213). Das Netzwerk wird dabei zu einer kulturellen Leitmetapher, mit der das mechanistische Paradigma der Maschine zugunsten eines „ganzheitlich“ vernetzten Denkens überwunden werden soll (Capra 1982). Friedrich und Biemann führen weiter aus, dass Netzwerke ab der Jahrtausendwende nicht mehr nur als Lösung der Krise der Moderne, sondern selbst als Teil des Problems angesehen werden. Heute wird der Begriff - wieder ambivalent - auch von Phänomenen des Verstrickt- bzw. Gefangen-Seins her gedacht, man denke an „Korruptions-Netzwerke“, „Schlepper-Netzwerke“ oder an problematisierbare Aspekte „sozialer Netzwerke“. In der alternativen Spiritualität scheint die Netzwerk-Metapher dennoch weiterhin ungebrochen positiv besetzt zu sein.

\section{Zusammenfassung und weiterer Forschungsbedarf}

In den untersuchten Texten von Fromm und Ferguson werden hoffnungsvolle Visionen eines ,guten Lebens“ formuliert, zu dem auch ein besseres gesellschaftliches Miteinander gehört. Die Leserschaft wird von ihnen ermutigt, am Prozess der Verwirklichung dieses Zieles mitzuwirken. Die Ergebnisse dieser Arbeit relativieren damit die These, wonach alternative Spiritualität gesellschaftliche Zusammenhänge grundsätzlich ausblende. Erich Fromms Anthropologie geht von einem primär isolierten Subjekt aus, welches diesen Zustand überwinden kann, sofern entsprechende gesellschaftlichen Bedingungen bestehen, an deren Herstellung es sich selbst beteiligen kann und soll. Marilyn Ferguson stellt mit der Netzwerk-Metapher eine Vision für die praktische Umsetzung vor. Der vorliegende Aufsatz verfolgt das Ziel einer Rekonstruktion, ideengeschichtlichen Einbettung und Diskussion ausgewählter zeitgeschichtlicher Selbstzeugnisse. Ausgehend von dem Befund, dass die Zuschreibung des Rückzugs in eine private Nabelschau sich nicht mit der Wahrnehmung und 
Darstellung der untersuchten Autoren und Autorinnen deckt, ergeben sich Perspektiven für künftige Forschungen, welche abschließend stichwortartig aufgezeigt werden.

Aus rezeptionsgeschichtlicher Sicht wäre der Einfluss von Ferguson und Fromm auf die alternativ-spirituelle Szene ihrer Zeit noch genauer zu klären und festzustellen, welche weiteren Positionen es in diesen Szenen bezüglich der Fragestellung gab. Hierbei können Quellen bis in die Gegenwart berücksichtigt und so Gemeinsamkeiten und Unterschiede zu den besprochenen „Klassikern“ herausgearbeitet und vor ihrem sozial- und kulturgeschichtlichen Hintergrund verortet werden.

Ein neuer Blickwinkel tut sich auf, wenn Fromm und Ferguson im Rahmen der Geschichte des linksalternativen Milieus ihrer Zeit gelesen werden. In diesem spielten nach Sven Reichhardt (2014) der Topos der Selbstverwirklichung und damit einhergehend der Ruf nach Authentizität eine zentrale Rolle. Das Ideal der Authentizität bzw. des wahren Selbst wurde dabei mit dem in diesem Milieu selbstverständlichen politischen Anspruch verknüpft. In Kontrast zu Theodor W. Adornos Diktum, nach dem es „kein richtiges Leben im Falschen“ (Adorno 1951/2003: 43) geben könne - und es daher zuallererst auf die Änderung der Gesellschaftsstrukturen ankomme - wurde auf eine „Politik der ersten Person“ gesetzt. Die Trennung zwischen Politischem und Privatem wurde, so der Anspruch, aufgebrochen und das Alltagsleben als revolutionäres Projekt verstanden.

Beim Subjekt und seiner Selbstbefreiung zu beginnen, bevor man eine gesellschaftliche Revolution beansprucht, war ein Credo, welches die klassische Gesellschaftskritik sozusagen umkehrte: Zuerst kam die Veränderung des Bewusstseins, dann die des gesellschaftlichen Seins (Reichhardt 2014: 873).

Statt sich auf die klassenlose Gesellschaft in weiter $\mathrm{Zu}$ kunft vertrösten zu lassen, wollte man das aktuelle eigene Leben neugestalten. Die „Graswurzelrevolution“ (Huber 1980: 57), die „Do-it yourself-Revolution“ (Ferguson 1982: 71) oder die „Revolution der Hoffnung“ (Fromm 1968) sollte die Gesellschaft von unten verändern. Das politisch verstandene Projekt der individuellen Selbstentfaltung wurde dabei mit dem Wunsch nach Gemeinschaftsbildung in einer als kalt und erstarrt wahrgenommenen Außenwelt verbunden (Reichhardt 2014). Auch Hero sieht die Gemeinschaft als die zentrale alternativ-spirituelle Institutionalisierungsform jener Zeit - heute hingegen sei die Szene marktförmig strukturiert. Die „neuen Heilsanbieter“ (Hero 2010: 135) würden in als Einzelunternehmen geführten Zentren ihre Vorträge, Beratungen, Therapiesitzungen und Ausbildungen anbieten und sich dabei ganz an den persönlichen Befindlichkeiten ihrer Klienten orientieren. Diesem Befund ist zum großen Teil zuzustimmen, allerdings sind auch heute alternativ-spirituelle Gruppen $\mathrm{zu}$ beobachten, welche sich als Teil eines „Netzwerks“ verstehen und ,persönliche und gesellschaftliche Transformation" anstreben. Phänomene wie etwa das Netzwerk Achtsame Wirtschaft, das Netzwerk Tiefenökologie, die Pioneers of Change, die Visionautik Akademie, das projectpeace, das an Ken Wilber orientierte Integrale Forum, die Politik des Herzens von Geseko von Lüpke oder zahlreiche Initiativen zu achtsamem Konsum und Ernährungsverhalten können als Ansätze zur Verwirklichung dieser grundlegenden Veränderungen verstanden werden. Diese Beispiele können als intermediäre Organisationen gelten, die im weiteren Sinne ,gemeinwohlorientiert“ sind und dadurch ,zivilgesellschaftliche Potentiale“ entfalten (Borutta 2005: 3).

Ob die in den Texten formulierten Ansprüche im täglichen Leben der Akteure des alternativ-spirituellen Milieus tatsächlich eingelöst wurden und werden, ist beim derzeitigen Forschungsstand - und vielleicht auch generell - nicht befriedigend zu beantworten. Empirische Studien zeigen jedenfalls, dass den Akteuren dieses Milieus Werte wie Altruismus und Solidarität überdurchschnittlich wichtig sind (vgl. z. B. Saroglou et al. 2005; Sarouglu \& Munoz-Garcia 2008; Farias 2008). Das äußert sich auch in einem entsprechenden sozialen und politischen Engagement (vgl. z. B. Engelbrecht 2009; Höllinger \& Tripold 2012). Der Frage nach der gesellschaftlichen Wirksamkeit relevanter Initiativen könnte mit Ansätzen des „social impact measurement" nachgegangen werden, wie sie bei der Bewertung und Optimierung gemeinnütziger Organisationen eingesetzt werden. Es besteht allerdings keineswegs Einigkeit darüber, was unter „social impact“ konkret zu verstehen ist (Choi \& Majumdar 2014) und welche Methode zu deren Messung angebracht wäre (Nicholls 2009; Kroeger \& Weber 2014). Auch Soziale non-profit Unternehmen - kirchennahe, nichtreligiöse und alternativ-spirituelle - stellt dies vor nicht geringe Herausforderungen, wenn es darum geht, den Wert des eigenen Handelns vor Stakeholdern darzustellen bzw. zu rechtfertigen (Ebrahim \& Rangan 2014). Weitere Forschungen könnten stattdessen eingehender analysieren, welche Ziele alternativ-spirituelle Netzwerke verfolgen, wie diese erreicht werden sollen und wie die Dimensionen des Individuell-Privaten und des Öffentlich-Politischen in diesem Kontext aufeinander bezogen werden und welche Zuschreibungen von individuellen und gesellschaftlichen Problemursachen und -lösungen sich daraus ergeben.

Interessenkonflikt: Der Autor bestätigt, dass kein Interessenkonflikt vorliegt. 


\section{Literatur}

Adorno TW (1951/2003) Minima Moralia. Reflexionen aus dem beschädigten Leben. In: Adorno TW (Hg.) Gesammelte Schriften, Bd. 4. Frankfurt a. M.: Suhrkamp.

Anhorn R, Balzerei M (Hg.) (2016) Handbuch Therapeutisierung und Soziale Arbeit. Wiesbaden: Springer.

Aristoteles (1999) Nikomachische Ethik. In: Flashar H (Hg.) Aristoteles, Werke in deutscher Übersetzung (6). Berlin:AkademieVerlag.

Assing-Hvidt E, Hansen HP, Iversen HR (2012) Glaube und Sinnorientierungen bei dänischen Krebspatienten in der Rehabilitation: eine taylorianische Perspektive. Spiritual Care 1:32-57.

Beck U, Beck-Gernsheim E (2002) Individualization. Institutionalized individualism and its social and political consequences. London: Sage Publications.

Beck U (1986) Risikogesellschaft: Auf dem Weg in eine andere Moderne. Frankfurt a. M.: Suhrkamp.

Bellah RN, Madsen R, Sullivan WM, Swidler A, Tipton SM (Hg.) (1996) Habits of the heart. Individualism and commitment in American life. Berkeley: University of California Press.

Berger P (1973) The social reality of religion. Harmondsworth: Penguin Books.

Bochinger C (1994) „New Age“ und moderne Religion. Religionswissenschaftliche Analysen. Gütersloh: Gütersloher Verlagshaus.

Borup J (2004) Zen and the art of inverting orientalism. Buddhism, religious studies and interrelated networks. In: Antes P, Geertz AW, Warne RR (Hg.) New approaches to the study of religion (1): regional, critical, and historical approaches. Berlin: De Gruyter. 451-488.

Borutta M (2005) Religion und Zivilgesellschaft - Zur Theorie und Geschichte ihrer Beziehung. Discussion Paper SP IV 2005-404. Wissenschaftszentrum Berlin für Sozialforschung.

Bruce S (2002) God is dead. Secularization in the West. Oxford: Blackwell.

Burkhart G (1998) Individualisierung und Elternschaft. Eine empirische Überprüfung der Individualisierungsthese am Beispiel USA und ein Systematisierungsvorschlag. In: Friedrichs J (Hg.) Die Individualisierungsthese. Wiesbaden: Springer.

Capra F (1982) The turning point: science, society, and the rising culture. New York: Bentam.

Capra F (1992) Das Tao der Physik. Die Konvergenz von westlicher Wissenschaft und östlicher Philosophie. Wien: Scherz.

Choi N, Majumdar S (2014) Social entrepreneurship as an essentially contested concept: opening a new avenue for systematic future research. Journal of Business Venturing 29:363-376.

Dobbelaere K (2002) Secularization. An analysis at three levels. Brüssel: Peter Lang.

Domagoj A (2011) Erich Fromm - ein jüdischer Denker. Jüdisches Erbe. Tradition. Religion. Berlin: LIT.

Ebrahim A, Rangan V (2014) What impact? A framework for measuring the scale \& scope of social performance. California Management Revue 56:118-141.

Engelbrecht M (2009) Die Spiritualität der Wanderer. In: Bochinger , Engelbrecht M, Gebhardt W (Hg.) Die unsichtbare Religion in der sichtbaren Religion - Formen spiritueller Orientierung in der religiösen Gegenwartskultur. Stuttgart: Kohlhammer. 35-81.

Farias M (2008) Holistic individualism in the age of aquarius: measuring individualism/collectivism in New Age, catholic and atheistic/agnostic groups. Journal for the Scientific Study of Religion 47:277-289.

Ferguson M (1982) Die sanfte Verschwörung. Persönliche und gesellschaftliche Transformation im Zeichen des Wassermanns. Basel: Sphinx.

Friedrich A, Biemann C (2016) Digitale Begriffsgeschichte? Methodologische Überlegungen und exemplarische Versuche am Beispiel moderner Netzsemantik. (Zitierdatum 25.01.2019), abrufbar unter https://www.inf.uni-hamburg.de/en/inst/ab/lt/ publications/2016-friedrich-biemann-digitale-begriffsge schichte.pdf.

Fromm E (1955/2003) Wege aus einer kranken Gesellschaft. Eine sozialpsychologische Untersuchung. München: dtv.

Fromm E (1956/2003) Die Kunst des Liebens. Frankfurt a. M.: Suhrkamp.

Fromm E (1960/1971) Zen-Buddhismus und Psychoanalyse. Frankfurt a. M.: Suhrkamp.

Fromm E (1968) Revolution der Hoffnung. Für eine Humanisierung der Technik. München: dtv.

Fromm E (1974) Anatomie der menschlichen Destruktivität. Stuttgart: Deutsche Verlags-Anstalt.

Fromm E (1974) Psychoanalyse und Ethik. Bausteine zu einer humanistischen Charakterologie. In: Funk R (1999) (Hg.) Erich Fromm Gesamtausgabe in 12 Bänden. Band II: Analytische Charaktertheorie. Stuttgart: dtv.

Fromm E (1976/2010) Haben oder Sein. Die seelischen Grundlagen einer neuen Gesellschaft. München: dtv.

Fromm E (2008) Ihr werdet sein wie Gott. Eine radikale Interpretation des Alten Testaments und seiner Tradition. München: dtv.

Hanegraaff W (1998) New Age religion and Western culture. Esotericism in the mirror of secular thought. Ney York: State University of New York Press.

Hardeck J (1992) Vernunft \& Liebe. Religion im Werk von Erich Fromm. Frankfurt a. M.: Ullstein.

Hero M (2010) Die neuen Formen des religiösen Lebens. Eine institutionentheoretische Analyse neuer Religiosität. Würzburg: Ergon.

Höllinger F, Tripold T (2012) Ganzheitliches Leben: Das holistische Milieu zwischen neuer Spiritualität und postmoderner WellnessKultur. Bielefeld: transcript.

Huber J (1980) Wer soll das alles ändern? Die Alternativen und die Alternativbewegung. Berlin: Rotbuch.

Jacoby R (1987) The last intellectuals. American culture in the age of academe. New York: Basic Books.

Johach H (2008) Erich Fromms humanistischer Sozialismus. Fromm Forum 12:34-47.

Kardorf von E (2016) Zur Transformation der Therapeutisierung und Psychiatrisierung des gesellschaftlichen Alltags: Auf dem Weg der (nicht ganz) freiwilligen Selbstoptimierung. In: Anhorn R, Balzerei M (Hg.) Handbuch Therapeutisierung und Soziale Arbeit. Wiesbaden: Springer. 263-298.

Klein-Landskron E (1990) Gesellschaftskritik und Lebenshilfe. Jahrbuch der Internationalen Erich-Fromm-Gesellschaft (1). Münster: LIT. 158-181.

Knoblauch H (1996) Soziologie der Spiritualität. In: Baier K (Hg.) Handbuch Spiritualität: Zugänge, Traditionen, interreligiöse Prozesse. Darmstadt: Wissenschaftliche Buchgesellschaft. 91-111.

Knoblauch H (2002) Ganzheitliche Bewegungen. Transzendenzerfahrung und die Entdifferenzierung von Kultur und Religion in Europa. Berliner Journal für Soziologie 3:295-307. 
Kolbe C (1986) Heilung oder Hindernis. Religion bei Freud, Adler, Fromm, Jung und Frankl. Stuttgart: Kreuz.

Kroeger A, Weber C (2014) Developing a conceptual framework for comparing social value creation. Academic Management Revue 39:513-540.

Krüger O (2010) L'homme machine oder die Überwindung und Vervollkommnung des Menschen im Posthumanismus. In: Assman J, Assman A (Hg.) Vollkommenheit. Archäologie der literarischen Kommunikation X. München: Wilhelm Fink. 107-129.

Lasch C (1980) Das Zeitalter des Narzissmus. München: Steinhausen.

Levinas E (2002) Totalität und Unendlichkeit: Versuch über die Exteriorität. Freiburg i.Br.: Karl Alber.

List E (1988) Flucht ins Paradies? Der soziale und historische Kontext von Therapiebewegungen und neuer Religiosität. Wege zum Menschen 7:237-248.

Luckmann T (1967/1991) Die unsichtbare Religion. Frankfurt a. M.: Suhrkamp.

Maasen S, Elberfeld J, Eitler P, Tändler M (Hg.) (2011) Das beratende Selbst. Zur Genealogie der Therapeutisierung in den „langen“ 70ern. Bielefeld: transcript.

Marcuse H (1969) Triebstruktur und Gesellschaft. Ein philosophischer Beitrag zu Sigmund Freud. Frankfurt a. M.: Suhrkamp

Martin D (1978) A general theory of secularization. Oxford: Blackwell.

McLaughlin N (2017) Who killed off Fromm's reputation in North America? Russell Jacoby's social amnesia and the forgetting of a public intellectual. Fromm Forum. 21:7-21.

Nicholls A (2009) 'We do good things, don't we?'. Blended value accounting in social entrepreneurship. Accounting, Organizations and Society 34:755-769.

Pickel G (2011) Religionssoziologie. Eine Einführung in zentrale Themenbereiche. Wiesbaden: Springer.

Pollack D, Pickel G (1999) Individualisierung und religiöser Wandel in der Bundesrepublik. Zeitschrift für Soziologie 28:465-483.

Pollack D \& Pickel G (2003): De-Institutionalisierung des Religiösen und religiöse Individualisierung in Ost- und Westdeutschland. Kölner Zeitschrift für Soziologie und Sozialpsychologie 55:447-474.

Pollack D, Rosta G (2015) Religion in der Moderne. Ein internationaler Vergleich. Frankfurt a.M.: Campus.

Pollack D (2003) Säkularisierung - ein moderner Mythos? Tübingen: Mohr Siebeck.

Remele K (2001) Tanz um das goldene Selbst? Therapiegesellschaft, Selbstverwirklichung und Gemeinwohl. Wien: Verlag Styria.

Rickert J (1986) The Fromm-Marcuse debate revisited. Theory \& Society $15: 351-400$
Runggaldier E (1996) Philosophie der Esoterik. Stuttgart: Kohlhammer.

Saroglou V, Munoz-Garcia A (2008) Individual differences in religion and spirituality. An issue of personality traits and/or values. Journal for the Scientific Study of Religion 47:83-101.

Saroglou V, Pichon I, Trompette L, Verschueren M, Dernelle R (2005) Prosocial behaviour and religion: new evidence based on projective measures and peer ratings. Journal for the Scientific Study of Religion 44:323-348.

Schneider-Flume G (1978) Leben dürfen oder leben müssen. Die Bedeutung der humanistischen Psychoanalyse Erich Fromms für die theologische Anthropologie. In: Reif A (Hg.) Erich Fromm. Materialien zu seinem Werk. Wien: Europa Verlag. 135-161.

Tschannen 0 (1991) The secularization paradigm. A systematization. Journal for the Scientific Study of Religion 30:395-415.

Taylor C (1996) Quellen des Selbst. Die Entstehung der neuzeitlichen Identität. Frankfurt a. M.: Suhrkamp.

Vivekananda S (1935) Hinduismus. Ansprache gehalten auf dem internationalen Religionskongress Chicago 1893. Zürich: Rascher.

Wilson B (1982) Religion in sociological perspective. Oxford: Oxford University Press.

Wohlrab-Sahr M (1997) Individualisierung: Differenzierungsprozess und Zurechnungsmodus. In: Beck U, Sopp P (Hg.) Individualisierung und Integration. Neue Konfliktlinien und neuer Integrationsmodus? Opladen: Leske und Budrich. 23-36.

\section{Biografische Angaben}

\section{Johannes Endler}

MA, geboren 1986 in Graz, Studium der Sozialen Arbeit an der FH Joanneum/Graz (Bachelor). Psychotherapeutisches Propädeutikum und tiefenpsychologische Weiterbildungen. Sieben Jahre Berufserfahrung als Sozialpädagoge (Jugendwohlfahrt) und Sozialarbeiter (Arbeit mit armutsbetroffenen Menschen). 2016 Abschluss Studium der Religionswissenschaft an der Universität Wien (Master). Mitglied im Arbeitskreis Religion und Medizin der DVRW. Dissertation über „alternativreligiöse Haltungen zum ungeborenen Kind“ in Arbeit. 